\title{
UN TEATRO REFUNDACIONAL: LA CONSTRUCCIÓN IDENTITARIA EN LA REAPERTURA DEMOCRÁTICA. LA PRODUCCIÓN FINAL DE FRANCISCO NIEVA
}

\author{
A REFUNDATIONAL THEATRE: THE CONSTRUCTION OF \\ IDENTITY IN THE SPANISH DEMOCRATIC RESUMPTION. \\ THE LATEST PRODUCTION OF FRANCISCO NIEVA
}

\author{
Federico LÓPEZ-TERRA \\ University of Sheffield / CCHS-CSIC \\ f.lopezterra@gmail.com
}

Resumen: El presente trabajo se propone el estudio la última etapa de la producción del dramaturgo Francisco Nieva. Largamente desatendida por la crítica, su puesta en relación con la «semiótica del cambio» que signó a la España de los años 80 permite arrojar nueva luz sobre esta producción.

Abstract: This article seeks to show the interrelation between the latest plays of the Spanish playwright Francisco Nieva and the so-called «semiotic of change» that characterised 80 s Spain. This study intends to shed some new light over this overlooked production and demonstrate its internal coherence.

Palabras clave: Francisco Nieva. Reapertura democrática. Teatro español.

Key words: Francisco Nieva. Democratic resumption. Spanish theatre. 
En el presente ensayo nos ocuparemos de la última etapa de la producción dramática de Francisco Nieva ${ }^{1}$. Desde un punto de vista estrictamente literario, creemos que la empresa vale la pena en una doble vertiente: por un lado, por tratarse de sus últimas producciones, lo que ya de por sí las constituye como cierto todo unitario y, por otro - aunque probablemente no se trate de una mera coincidencia-, por ser la parte de su obra que menor atención ha recibido por parte de la crítica, a pesar de tratarse de uno de los autores más importantes de la dramaturgia española del siglo XX.

Por otro lado, creemos que esta producción merece una atención especial en virtud del contexto sociopolítico que la enmarca. Desde este punto de vista, estas obras, que normalmente caen dentro de un saco de difícil clasificación, revelan una unidad asombrosa. La continua vuelta sobre sus textos tanto en forma de correcciones, reescrituras e incluso como continuación de obras comenzadas e interrumpidas años antes, hace difícil una clasificación cronológica. Su estética particular y sus propios criterios, lejos de allanar el camino, dificultan aún más cualquier intento (Barrajón, 1994: 5-6). Por otra parte, el criterio eminentemente estético con que se ha solido clasificar la producción nieviana esconde su fuerte vinculación con un período determinado de su producción, el de la década del 60. La aplicación del mismo a las obras gestadas en los años 80 ha inducido, en nuestra opinión, al error de considerarlas en su mayor parte retazos marginales de una producción más amplia. En el presente trabajo postularemos la relativa autonomía de estas obras e intentaremos explicar cómo el encuadre dentro del periodo democrático da cuenta de un cambio que no es tan solo estético.

\section{LA PRODUCCIÓN FINAL DE FRANCISCO NIEVA EN EL CONTEXTO DE LA REAPERTURA DEMOCRÁTICA}

En adelante, intentaremos demostrar que estas obras presentan una unidad que se explica fundamentalmente a la luz de la semiótica social que proponen, pero que, y por consiguiente, configuran una estética particular que no puede ser explicada a partir de su producción anterior.

Desde un punto de vista socio-cultural, estas obras se vinculan al proceso de transición y reapertura democrática vivido por España en la década

1 La producción dramática de Francisco Morales Nieva (Valdepeñas, Ciudad Real, 1924) es amplia pero no extensísima. Su obra se reparte desde los años 50 hasta la década del 80, siendo su período más fecundo el de la década del 60, que se corresponde con su exilio voluntario en Francia. 
del 80, más precisamente a partir de 1982, año clave —y consensuado- de la reapertura democrática, una vez superado el periodo denominado de «transición», que se cierra con dos grandes acontecimientos de gran valor simbólico: el golpe fallido del coronel Tejero, el 23-F del 81, y la victoria del PSOE en las elecciones de 1982.

El proceso de democratización nos interesa como fenómeno sociocultural por varias razones. Por un lado, porque ya no se inviste al Estado del poder de ejercer una tutela moral sobre los ciudadanos y, por otro, porque supone la restitución de la sociedad civil a un lugar protagónico dentro de la esfera pública ${ }^{2}$. Es precisamente de sus tradiciones de donde se extraerán los códigos que conformarán la nueva vida democrática. En este proceso de «reinvención» de la identidad Pérez-Díaz (1993: 19) señala que:

la invención de aquella nueva tradición supuso la elaboración de un nuevo lenguaje cultural... y, por otro lado, la reconstrucción de la memoria colectiva, con un nuevo entendimiento de la Historia de España, y con la elaboración de nuevos mitos edificantes.

Esta restitución no se gesta de un día para otro, sino que implica un proceso, en sucesivas etapas, de recuperación de espacios simbólicos y de significados. En España, estas corresponderían a la liberalización del régimen franquista que se produce principalmente a partir de la década del 50 de manera progresiva y que puede verse reflejada a nivel filosófico-literario en la disputa que mantuvieron Sastre (1960a: 1-2 y 1960b: 1-2) y Buero Vallejo (1960: 1-6) en torno al «posibilismo» en las páginas de Primer Acto ${ }^{3}$. A partir de entonces, la nueva vida en democracia supondría el tránsito por los estadios de transición, consolidación e institucionalización ${ }^{4}$.

Estas etapas del proceso democrático tienen su correspondiente dentro de la conformación de las nuevas redes de significación: en primer lugar, una instancia de redefinición de sentidos, más tácitos que explícitos; una segunda instancia de apropiación de sentidos y negociación de los mismos; y una última que correspondería, a su fijación, entendiendo

2 Para una discusión más amplia sobre el concepto de sociedad civil en sentido amplio y restringido, véase Pérez-Díaz (1993, 1997).

3 Para el fenómeno de «liberalización» en los procesos de reapertura democrática véase Huntington (1994).

4 Para una descripción más detallada de las etapas de transición, consolidación e institucionalización para el caso español, véase Pérez-Díaz (1993). Para una discusión más detallada sobre el proceso de consolidación en los años '80, véase Villoria Mendieta (1999) y Wynants (2006). 
que estos son válidos como rectores de la vida en sociedad y que, por consiguiente, pueden ser sometidos a un proceso de legitimación simbólica (Pérez-Díaz, 1993: 44).

La producción que hemos seleccionado como corpus de este trabajo remite a esta última instancia en el proceso de significación, que coincide con la España de la institucionalización.

A este periodo corresponden ocho de las obras de Nieva que, si bien podrían parecer pocas a primera vista, acaban constituyendo casi la tercera parte de su producción dramática total. El grupo de obras que consideramos el núcleo capital de este periodo estaría constituido por: Te quiero, zorra, Corazón de arpía, No es verdad (todas ellas de 1987) y, un poco más colateralmente, Carlota Basilfinder (1987) y Catalina del demonio (1988)5.

Sin duda, existe continuidad en la línea estética del dramaturgo entre estas obras y las anteriores. Sin embargo, la producción más reciente propone nuevos tópicos y radicaliza otros, dando así un nuevo sentido general a estas últimas producciones. El estudio de estas piezas a la luz de obras consagradas como las reóperas, ha conducido, desde nuestro punto de vista, al error de descuidar un aspecto esencial de las mismas: su unidad y sistematicidad.

La propia concepción de Nieva sobre la inmediatez del fenómeno teatral - «un fenómeno muy efímero, después de un estreno todo se olvida y lo que queda es lo publicado»- (Nieva, 2007), refuerza la validez de emprender la exégesis de los referidos textos a la luz de una reconfiguración semiótica particular que diferencia claramente a la década del 80 en España de las precedentes. Pero además, parecería tratarse de una producción «cerrada», según el propio autor, al declarar que «ya no se siente con fuerza para crear nuevas obras, pero sí para reescribirlas, repasarlas y modificarlas» (Nieva, 2007). De hecho, el fenómeno de reescritura es prueba fehaciente de una voluntad de actualización permanente del texto, solo posible en la medida en que en la raíz del mismo se entienda como mudable la cosmovisión que le da sentido.

Desde este supuesto, nos moveremos, a continuación, hacia la descripción de algunos de los elementos constituyentes de la producción dramática de estos años a la luz de las nuevas necesidades de significación de la

5 De aquí en adelante citaremos las obras por su acrónimo ( $T Q Z, C D A, N E V, C D D$ y $C B)$ y número de página según la siguiente edición: Peña (2001b: 1241-253, 1255-276, 1279-301, 1323-333 y 829-68, respectivamente). 
España de la reapertura democrática, sea como síntomas o como consecuencia de las mismas. De ahí que la estética propuesta en estas obras pueda ser entendida también como representativa de toda una tradición literaria del periodo.

\section{ALGUNAS CLAVES DRAMÁTICAS: EL TEATRO EN REPRESENTACIÓN DEL NUEVO ACTOR SOCIAL}

\subsection{La metamorfosis y la irrupción de lo fantástico}

La metamorfosis se descubre como uno de los rasgos más significativos y recurrentes de las obras de este periodo. Próxima a la estética de la animalización y de los personajes «entreverados» tan típicos de Nieva, la metamorfosis ocupa un lugar claramente diferenciado.

Podría intentar trazarse una cronología de la animalización desde estos personajes comenzando por Blanche, la protagonista de $N E V$, quien nunca llega a revelar en escena una verdadera metamorfosis más que en forma de indicios. Zoé (TQZ) experimenta ya una verdadera mutación con su rabo de zorra, que tampoco se revela en escena, pero es referido explícitamente. La culminación sería el personaje de Dirce $(C D A)$ donde la hibridación (que no metamorfosis) queda manifiesta sobre el escenario.

En el caso de estas obras, lo fantástico - la metamorfosis - se oculta al resto de personajes como prueba de que forma parte de una serie de fenómenos que no encuentran explicación y, por consiguiente, no podrían integrarse de manera natural dentro del mundo en el que suceden. Para ello, existe siempre algún personaje que funciona como parámetro de la «normalidad» y que actúa como contrapunto para evidenciar esta desviación. A diferencia de lo raro, que tiende a integrarse planamente y sin conflicto con la lógica interna generada por el texto, lo fantástico en estas obras pone de manifiesto el principio de contradicción que pondrá en escena, al menos, dos epistemologías encontradas. Esta bipolarización comienza a través de la ininteligibilidad, el descrédito y la sorpresa que genera el suceso en los personajes-antagonistas:

ZOÉ.-Me he convertido en una zorra... ANAÍS.-iNo es posible! (TQZ: 1242-43).

En el caso de $C D A$ no hay metamorfosis, ya que Dirce es desde un comienzo - y desde siempre - una arpía. Por este motivo podría entendérsela 
más próxima a los personajes híbridos de Nieva y, por consiguiente, incluirla dentro de la categoría de lo aberrante. Sin embargo, como señalábamos más arriba, el hecho de que en la misma obra aparezca uno de estos personajes «entreverados» hace que su lugar sea otro. La arpía, a diferencia de estos seres, no es un ser ambiguo, sino todo lo contrario. Además, aunque en este caso no opere ninguna trasformación, lo fantástico en sí es la posesión de Dirce por parte de Luciano. Y si bien el mundo mítico de la obra tiende a la normalización de su aparición, lo cierto es que Dirce sigue siendo un personaje extraño, aun dentro de su propio mundo mitologizado. Esto, al igual que en los casos anteriores, se expresa mediante la sorpresa, en este caso de Creonte:

LUCIANO.-Me casé con una arpía...

CREONTE.-¿Qué me dices? ¡Una arpía de verdad! (CDA: 1260).

O también en el caso más ambiguo presentado en $N E V$, donde nunca termina de quedar claro hasta qué punto se ha operado una verdadera metamorfosis en la protagonista, Blanche:

BLANCHE.-Estás fuerte porque te alimentas como ellos [los lobos]. Lo sé. Oh, Eric, llévame. Pero llévame contigo para siempre, sácame de aquí de una maldita vez.

ELIN.-iQué escándalo! Así es como te despojas de todo pudor... Pero ¿qué extraño veneno se ha inculcado en ti? (NEV: 1285).

Es interesante observar que, salvo en el caso de Dirce en $C D A$, la metamorfosis no se muestra en escena, sino que se sugiere mediante elementos más o menos indiciales; la verdad última nunca queda al descubierto ante el público. Tal ocurre en $T Q Z$, donde se apunta en una acotación: «Zoé se mantiene de espaldas, gimiendo contra la pared y bajo su camisón, que deja media pantorrilla fuera, alardea fecundo y airoso el rabo de su condenación» (TQZ: 1249).

El procedimiento de ocultación recuerda al principio del drama clásico, según el cual la representación de «lo trágico» quedaba fuera de escena. La filiación de este recurso con la cuna de la tradición dramática de Occidente entra en consonancia con el gusto de Nieva por incluir elementos primitivos como forma de recuperar la esencia ancestral del drama (Pérez Coterillo, 1975: 37-8). Sin embargo, este vínculo va más allá de la mera relación con la tradición genérica. Tal y como afirma Linda Hutcheon al referirse a la poética de la posmodernidad, «the past is always placed critically —and not 
nostalgically — in relation with the present» (Hutcheon, 1988: 45); pero es que, además, en la obra de Francisco Nieva, el uso del pasado adquiere un valor instrumental fundamental para repensar el presente. Así, la tragedia no vista coloca al público ante el dilema de decidir si comulgar o no con la realidad que se le presenta en escena, situando al espectador en una frontera de ambigüedad e incertidumbre con la que al autor le gusta jugar. Esta característica del teatro de Nieva es tal vez una de las más importantes en el periodo de reapertura democrática. La ambigüedad conduce al espectador a esta zona difusa donde la única determinación posible está en su papel activo ante la realidad que se le presenta. Nieva logra conjugar una profunda crítica social sin ni siquiera una alusión directa, pues como apunta Komla Aggor (2009: 99):

El objetivo político de Nieva no es agitar al público contra un objetivo concreto como podría ser el franquismo, sino criticar y rebatir las fuentes de dominación cultural represiva en su sociedad mediante un mecanismo paródico y juguetón a la vez.

En este caso, lo que se establece es una frontera entre el espacio escénico propiamente dicho y el del espectador, para quien lo fantástico nunca se evidencia, dejando margen para la duda y la especulación. Tal es lo que ocurre en $N E V$ cuando luego de haber sido planteada en la primera escena la relación de los jóvenes con los lobos, como una ficción compartida por los tres personajes, se abre la segunda escena como sigue:

Blanche borda en un bastidor. Al cabo de un rato, deja el bastidor y saca de entre sus faldas un pañuelo sanguinolento y, con aire furtivo, se lo lleva a la boca, tratando de despedazar un trozo de carne cruda oculta en él (NEV: 1282).

Esta segunda escena parece contravenir la primera, proponiendo la posibilidad de que la pretendida ficción sea real. Sin embargo, el gesto es lo suficientemente ambiguo como para convencernos de que tal cosa sea así. La intromisión que propone la obra en la esfera privada de la escena está limitada por el acceso parcial a la información de que dispone el público ${ }^{6}$. El acceso a la verdad última será, por lo tanto, siempre conjetural. El espectador ve la

6 Para un análisis más detallado de la categoría dramática de «visión», véase el capítulo homónimo en García Barrientos (2001). 
pieza desde la perspectiva de Elin y por tanto es desconocedor, como él, de los planos de ficción o realidad que construyen Blanche y Eric a su alrededor.

La utilización de este recurso plantea una subdivisión entre el espacio público y el espacio privado, donde el fenómeno teatral supone la penetración de lo público en la esfera de lo privado, pero cuyo acceso parcial evita que sus límites se desdibujen por completo. Se dan a la par dos fenómenos opuestos: la publicitación y la salvaguarda del espacio privado, paradoja que pone al descubierto la compleja relación entre espacio público y privado en la reconfiguración de los espacios simbólicos que se produce a partir de la reapertura democrática, cuando, precisamente, se intenta reconstruir el concepto de lo privado, hasta entonces mellado por la intromisión - muchas veces más simbólica que real- del poder estatal en la vida cotidiana. El fenómeno queda bien descrito por Imbert (1990: 32): «[L]a privacidad está cada día más amenazada en tanto que espacio y, por eso, es reivindicada como valor. En general de lo que se trata es de proteger la esfera privada de las injerencias del Estado», y entiéndase por Estado, en la concepción dramática propuesta, cualquier centro hegemónico de dominación.

Pero, por otra parte, nos enfrentamos a la paradoja de la exposición de esa privacidad como reivindicación de otro derecho: la liberación del cuerpo y del discurso del control estatal. De ahí que sobrevenga al mismo tiempo en la España postfranquista el fenómeno del «destape», caracterizado por el mismo Imbert (1990: 33) como la «liberación de los tabúes relativos al cuerpo y 'boom' informacional que acompaña el 'levantamiento' de la prohibición. Esto puede ser también reivindicar el valor de lo privado en la legislación social», en lo que el teatro y el cine como formas de representación de masas cumplieron un papel preponderante.

\subsection{La metamorfosis como transformación}

Por encima de su valor ficcional o dramático, la metamorfosis entendida dentro del fenómeno comunicativo que supone la representación pone en escena una semiótica de la transformación especialmente sugerente. En todas estas obras la transformación entraña una de-formación, o al menos una desviación en un sentido más amplio, y tiene este valor en tanto es visto como maldición, condena o castigo divino. En referencia a su transformación comenta Zoé: «[M]i estigma es todavía mayor» (TQZ: 1242).

No son estos personajes los que optan por la transformación, sino que en una concepción bastante más clásica, es el destino el que se impone. El he- 
cho de que antes o después acaben aceptándolo de manera gustosa - a diferencia del héroe clásico - no debe hacernos olvidar su condición de condenados.

Como desviación, los dramas comienzan instaurando un statu quo: la normalidad, a diferencia de lo que ocurre en otras obras, es un mundo plagado de convencionalismos y extremadamente codificado. En este contexto, cualquier desviación será aberrante y, por consiguiente, censurable.

Por esto mismo, es interesante observar que este paradigma es gradualmente subvertido, a medida que la metamorfosis deja de ser una de-formación y se vuelve una trans-formación, trocándose de condena en transgresión. En esta cadena es donde se inserta toda la serie de modificaciones semánticas, a saber: la maldición se vuelve bendición, el mal se trueca en bien, y así sucesivamente. La inversión de valores es una constante del teatro de Nieva como teatro del mal, pero solo en estas obras queda patente la inversión como proceso, como transformación que queda incluida dentro de la propia obra ${ }^{7}$.

Estas son obras en las que a los personajes les sucede algo anormal, y esta anormalidad consiste precisamente en una transformación que ocurre - o se descubre - en escena. Lo importante, entonces, no es tanto una semiótica de lo bestial, sino de la mutación como transformación. Se trata de la diferencia entre una semiótica «estática» y otra procesual donde los personajes se vuelven aberrantes. En todas las obras de Nieva los protagonistas (¿héroes?) viven una transformación de principio a fin. La evolución - la mayor parte de las veces entendida como involución - es parte constitutiva de su heroicidad, sin que por ello medie la paradoja o la contradicción. En las piezas anteriores al 80, la transformación se puede caracterizar básicamente a través de dos constantes: se trata de una trasformación moral, no física, y voluntaria.

En estas otras, por el contrario, la trasformación es o bien impuesta, como en el caso de Zoé, o bien producto de un cierto determinismo. La metamorfosis no sería un simple cambio, sino la propia metáfora del cambio, y en este sentido no tiene - ni puede tenerlo- un significado único. Y no se trata solo de un fenómeno de recepción, sino que tampoco desde la enunciación podría venir asignado un sentido unívoco, pues no hay un referente

7 Para un estudio sobre las vinculaciones de la obra de Nieva con el teatro de Artaud y la obra de Bataille, véase Aggor (1998). 
oculto o simbolizado más que el cambio en sí, de lo que se deriva la infinidad de posibilidades interpretativas que sobre él recaen.

Lo que se observa como constante significativa en este proceso es la renuncia a la humanidad - como naturaleza y como género- en pos de un acercamiento a una forma de animalidad. Esta aproximación bien podría verse como involución, sin embargo, ya hemos visto cómo funciona la resemantización de lo monstruoso hacia lo positivo. La evolución a lo largo de la obra cambia el signo negativo de lo bestial por el reverso primigenio, ancestral. Dice Viller: «Este era tu maravilloso misterio... Ahora descubro que el verdadero amor es divinamente bestial» (TQZ: 1250).

Acorde con la tendencia de Nieva a la utilización de modelos arquetípicos del imaginario colectivo, se induce al retorno de una lógica irracional y a la liberación de principios de orden convencional (Pérez Coterillo, 1975: 39).

\subsection{La construcción de lo marginal: el arcano femenino y la sociedad civil}

Uno de estos arquetipos es el que Nieva denomina «principio femenino», al que vincula con la recuperación de «un sentido positivo, tenaz y renaciente, germinal, creacional, rebelde», resignificado en el periodo en cuestión por todo lo que supone como principio refundacional (Nieva, 1980: 113). Al respecto señala Aggor (2000: 438-39):

Nieva's project to exalt the «ex-centric» finds its most forceful expression in the removal of traditional gender gaps. In Nieva's drama, women take the center stage. They are strong, rebellious, triumphant.

El protagonismo femenino es, efectivamente, uno de los rasgos más sobresalientes de estas piezas. La mujer ocupando el centro funciona como perspectiva desde los márgenes, es decir, son obras en las que la acción se desarrolla desde una posición ex-céntrica en su sentido más literal, y es sobre la figura de la mujer sobre la que se construye esta acción desplazada. Esta semiótica del margen entraña una particular cosmovisión desde un lugar sumamente significativo. Uno de los desafíos más importantes para la España de la década del 80 es la restitución de las minorías excluidas y la constitución de la denominada «sociedad plural» (Imbert, 1990: 190-191). Esta construcción implica necesariamente la aceptación más o menos explícita de la existencia de un centro de poder representante del modelo social 
y, por consiguiente, la aceptación también de todo otro grupo marginal $-\mathrm{y}$ marginado-, cuya constitución depende del lugar que ocupe con relación al considerado «centro», y su adecuación o no al modelo que este imparte.

Por consiguiente, estas obras constituyen un procedimiento de corrimiento hacia «las afueras» como forma de inclusión. Pero lo realmente importante es que esa inclusión se realiza desde el propio margen como centro y no desde una perspectiva hegemónica que habilita la inclusión del otro. Esta no es una obra para el otro, sino que se trata de las obras del otro, quien ocupa los lugares «centrados» de significación.

Este procedimiento sigue la lógica de la inversión a la que hacíamos referencia más arriba. En las obras anteriores al 80, el protagonista, representante del mundo «convencional», acaba adentrándose en la lógica paralela que propone este mundo degradado en el que voluntariamente decide perderse, contraviniendo así la marca negativa de esta perdición ${ }^{8}$. La cooptación del ser representante del mundo del «deber ser» se vuelve un mecanismo compensatorio con respecto a la violencia que representa la marginación. Por eso, lo marginal se representa de manera colectiva, en oposición a la individualidad del ser «otro».

En las obras posteriores al 80 , se invierte esta perspectiva y, aunque mantenga un sentido análogo, se construye en cierta medida como su negativo, pues el mundo representado es el de los convencionalismos, y aquí es el personaje protagónico el excluido o marginal desde el punto de vista social. La aceptación final gustosa de esta condición segregada acaba reivindicando la posición de exclusión, y no condenándola. En este sentido, no parecería erróneo afirmar que acaban por transformarse también en obras de la integración, a pesar de estar construidas sobre el principio de la exclusión, en la medida en que no se proponen perpetuarla de manera reivindicativa $o$ compensatoria, tal como ocurría en las obras anteriores al 80.

Otro rasgo distintivo de estas piezas con relación a la producción precedente es que este mundo reglado constituye un personaje en sí mismo: la sociedad civil.

La construcción de este personaje es compleja, atravesada por aparentes contradicciones y signada por la ambigüedad. En estas obras, es el conjunto social el que pertenece a la esfera de la normalidad, mientras que el individuo (el protagonista) es el relegado. Esta lógica provoca que, en un primer

\footnotetext{
8 Para un estudio comparativo, véase el estudio preliminar de A. González (1980).
} 
nivel, la sociedad civil se presente como grupo de pertenencia al que es deseable adscribirse. La sociedad civil y la posibilidad de integración a la misma representan, con matices, una idea común como sugiere Elin en $N E V$ : «Un doctor demostró que mi razón no había sido presa de aquel desvarío impuesto por los otros dos y me devolvieron a la vida civil» (NEV: 1289). Sin embargo, la relación con las protagonistas marginadas es un poco más compleja y es allí donde comienza el corrimiento del concepto hacia otras esferas de significación. En primer lugar, porque este concepto se construye de manera dialéctica y por oposición a estos personajes disidentes, convirtiéndose gradualmente de simple referente en su antagonista:

CREONTE.-Una de esas quimeras [Dirce] que bajan del monte a devorar las sobras de los banquetes, que siembran el desorden y la suciedad, que maldicen y estorban la vida civil de las personas, que se mofan de las buenas costumbres (CDA: 1244).

De este modo, esta entelequia va definiendo lentamente un sentido contrario y su carácter centralizante adquiere un cariz negativo de dominación sobre el individuo. El texto actúa de manera dinámica, realizando un corrimiento en paralelo que acercando al personaje marginado hacia el centro distancia, por oposición, el centro hacia el margen. Creemos que es precisamente esta relación dialéctica la que otorga a la sociedad civil el estatuto de personaje en tanto se construye en relación al protagonista. Pero al tiempo que adquiere de este su valor agentivo - actancial diríamos en términos estructuralistas- pierde su valor hegemónico extratextual. Al pasar a formar parte de la matriz textual «narrativa» mediante esta lógica transaccional pierde su estatuto central en favor de la centralidad del texto, es decir, el valor protagónico como categoría central del relato. La propia matriz textual desliza la díada central/marginal de un polo hacia el contrario. De este modo, los recursos por los que se construye en el texto la noción de marginalidad y de centralidad recaen tanto en el uno como en el otro hasta acabar por invertir estos valores.

Los protagonistas nievianos se encuentran sistemáticamente fuera de esta «vida civil». Por este motivo, no sería extraño vincular a la sociedad civil con una nueva forma de opresión sobre las individualidades, de ahí que se encuentre asociada en el discurso a grupos representantes del poder central como lo es paradigmáticamente la Iglesia que, al igual que este nuevo 
modelo de sociedad, ha cumplido un papel ambiguo en el proceso de la transición hacia la vida democrática ${ }^{9}$ :

ANAÍS.-Condenada en vida deshonestamente. Así serás rechazada por la Iglesia y por toda la sociedad civil (TQZ: 1244).

No se trata aquí de realizar una correspondencia inequívoca y atemporal entre sociedad civil e Iglesia. La sociedad civil, con este peso hegemónico, es una realidad que, a diferencia de la Iglesia, es nueva. Sin embargo, en este corrimiento hacia el centro, la sociedad civil acaba siendo lo mismo que la Iglesia era para el franquismo, es decir, el centro de poder social no estatal. La correspondencia no es automática, sino procesual: la sociedad civil no es como la Iglesia, sino que se vuelve como la Iglesia y, lo interesante, es que lo hace en el propio texto frente a nuestros ojos. De este modo Nieva consigue denunciar los peligros de los centros hegemónicos de poder y pone sobre la mesa uno de los principales problemas de la reapertura democrática española: la integración de las minorías (Imbert, 1990: 55-6).

La visión de la nueva sociedad civil puede quedar bien ejemplificada en las palabras con las que Gastón Villier invita a Zoé a vivir juntos y aislados del mundo:

VILLIER.-[Y]o no te dejaré jamás formar parte de una corporación... Allá los otros. Si bien lo piensas, todas las pasiones y las demasías del placer son una vergüenza. Claro, para los demás, que no viven con bastante intrepidez. Pues finjamos, querida; ocultémoslo y, al mismo tiempo que lo cargamos de culpa, lo valoraremos mejor (TQZ: 1252).

La idea que maneja de corporación nos parece bastante ejemplificativa de una concepción social centralizante y desde la cual se organizan los códigos de inclusión y exclusión. De ahí que la compensación funcione nominando como «otros» a los que en sentido propio serían los verdaderos nosotros. Apunta Komla Aggor (2000: 437):

In his resolute engagement with the Other, he undermines authority and glorifies the marginalized. [T]hrough their actions, they subvert the order under which they have been condemned. In this sense, the theatrical art becomes a political instrument of protest.

\footnotetext{
9 Véase al respecto Ysàs (2004), el capítulo titulado «La disidencia eclesiástica».
} 
Sin lugar a dudas, este recurso acerca al lector/espectador hacia los personajes excluidos. Sin embargo, Nieva elude sistemáticamente cualquier forma mecánica de empatía, conduciendo de la mano personajes y situaciones hacia la frontera poco tranquilizadora de la ambigüedad. Como sostiene en su «Pequeña teoría sobre un teatro histórico-didáctico», «la obra ha de asentarse en referencias culturales y de conocimiento histórico y no ha de pretender ser totalizadora y orgullosamente universalista» (Nieva, 1976: 14).

No olvidemos que, luego de la muerte de Franco, la sociedad civil es uno de los estandartes sobre los que construir la transición hacia la democracia, paradigma de lo democrático en oposición a la sociedad de tipo castrense que caracterizó al régimen anterior. Por este motivo, y por la fuerza conceptual y simbólica que suponía, se trata de un concepto - más propiamente un nuevo recorte social- en tensión y en pugna por los diferentes actores políticos y sociales, y el núcleo de acción en el que, a partir de ahora, se gestarán los sentidos que orientarían la nueva vida en sociedad (Pérez-Díaz, 1993: 58). Por esta razón, el simple hecho de que aparezca en la obra de Nieva en forma de referencias da cuenta de la ligazón del texto a un contexto determinado y del interés del autor por vincular y actualizar sus obras a la luz de la semiótica social del periodo. Pero explica, además, el valor agentivo que le otorga en sus obras a este «personaje» y la construcción compleja y ambigua que hace del mismo; construcción de la que no rehúye, sino que, por el contrario, acentúa. Se trata de la elusión del «universalismo», donde se cifra una de las claves más importantes de la ética del teatro de Nieva.

\subsection{El lenguaje como esencia de la historia y del cambio}

Está claro que el lenguaje teatral, entendido en un sentido amplio, ha sido siempre para Nieva una de sus principales preocupaciones en su revaloración de la escena y su concepción del denominado «teatro total» ${ }^{10}$. Dentro de los muchos lenguajes que este incluye, la materia lingüística tiene un sitio de privilegio.

El lenguaje tiene un lugar destacado en el proceso mismo de transformación, ya que, como propondremos, la metamorfosis es básicamente un fenómeno discursivo. El ejemplo paradigmático es el de Zoé, la prostituta que se metamorfosea en zorra. La transformación no es arbitraria, sino que se basa en la coincidencia entre dos significantes. Este fenómeno de

10 Para una definición ejemplar del propio autor véase Peña (2001a: 173). 
homonimia vincula a su vez a sus referentes y, por consiguiente, la prostituta - vulgarmente «zorra»— se transforma en el animal, de modo tal que la identidad verbal se vuelve referencial. Esta se refuerza en la obra cuando se produce una confusión lingüística entre ambos significados del término, donde - tanto en la lengua como en la realidad - una (palabra y personaje) es ambas cosas a la vez:

ZOÉ.-[Q]uiero que te vayas, Anaís. ;Vete! Prefiero suicidarme con mi secreto.

ANAÍS.-Pero ¿qué secreto, si está a la vista? Si tú misma te sientes zorra yo te lo admito y no voy a pedirte un certificado...

ZOÉ.-No entiendes nada... Escucha y créeme: me ha crecido un rabo, una cola peluda y larga al extremo de la rabadilla, un verdadero jopo de zorra (TQZ: 1243).

De esta manera, la «zorra» puede volverse zorra porque en la raíz del fenómeno ambas identidades son indisociables.

En $C D A$, aunque no existe metamorfosis en sentido estricto, la coincidencia lingüística también funciona uniendo ambos significados:

LUCIANO.-Me casé con una arpía.

CREONTE.-Bah, como yo. En el fondo, la llorona de mi mujer es una arpía (CDA: 1260).

El valor asignado al lenguaje en estas obras es mucho más sintomático que en la producción anterior, aquí se trata de una verdadera filosofía de la palabra. Solo entendiendo que la verdadera esencia del mundo está constituida por el lenguaje es como puede presentarse la metamorfosis como un fenómeno de lengua.

También es lingüística la metamorfosis de Blache en $N E V$, donde la ficción que crean los personajes para entretenerse acaba transformándose en realidad. Se trata de una historia narrada que por fuerza de repetirse acaba cooptando la realidad o, mejor dicho, justamente por tratarse de una materia narrada es que puede volverse real.

Vinculado a la construcción identitaria discursiva se encuentra también el reverso de la enunciación: el silencio. Contrapartida que actúa como hemos visto en la complejización de la construcción de la realidad y en la ambigüedad intencional de los textos nievianos.

El silencio supone el reducto fundamental de la identidad de estos personajes. En $C D D$ los personajes protagónicos de Gorro y Catalina se cons- 
truyen mediante su vinculación con el mismo. Lo no dicho, lo no proferido, no es más que la destrucción de la propia memoria entendida como registro histórico, y como tal, potencialmente reescribible. De ahí que cuando Gorro hable finalmente, interrogado por Catalina sobre la culpabilidad de los asesinatos, declare:

CATALINA.-Habla, habla de una vez. ¿Quién lo hizo?

GORRO.-Tú. Sola. Solo tú (CDD: 867).

$Y$ es que el mismo silencio que significa ausencia (de culpa, en este caso) para Gorro, se carga de significado para Catalina. Así, el silencio es también generador de discurso y, dentro de la obra, del propio ser; más concretamente, del ser trágico, ya que Catalina es, y es víctima, porque Gorro calla.

También en $C D A$ y en $T Q Z$ el silencio está presente como ocultación. Lo no dicho cumple la función de preservar la identidad del personaje que calla $\mathrm{y}$, en este sentido, funciona como preservación identitaria frente a lo que es considerado como impropio. Así ocurre con Villier, quien oculta su identidad para poder acceder a un mundo que de otra manera le estaría vedado, o como en el caso de Dirce, que calla para poder manipular a quienes creen manipularla. El silencio es protector y restitutorio en cada caso.

En $N E V$, como en $C D D$, también podría hablarse de una retórica del silencio que pretende difuminar los límites entre realidad y ficción, verdad y mentira, reescribiendo así la «historia oficial».

Silencio y olvido se vuelven prácticamente uno, vinculados a la preservación de la identidad. Callar es salvarse, pero al mismo tiempo el silencio condena a los olvidados, en la medida en que memoria y palabra van de la mano. La presente consideración se encuentra directamente vinculada a la reconsideración de la Historia como relato, propia de la reapertura democrática, al tiempo que deja en evidencia la importancia de considerar en estas construcciones el valor de lo que se calla. A propósito apunta González Duro (2005: 140): «El pasado franquista no había sido tanto dominado, como silenciado».

El valor trascendental que adquiere la memoria y su función ética en la definición de la identidad se expresa en boca de Anaís:

ZOÉ.-Una se emborracha, pierde la conciencia. Eso no es una falta. ANAÍS.-Siempre es una falta la falta de memoria (TQZ: 1245). 
En lo que se refiere al vínculo establecido entre la retórica propuesta en las obras y la semiótica social del periodo, parece clara la importancia del peso que jugó en la transición el pacto implícito de silencio y de olvido voluntario:

Para que no hubiese solución de continuidad en la transición desde el régimen franquista a la democracia... fue preciso olvidar que Franco había existido... Los españoles tuvieron que negar su reciente y ominoso pasado histórico (González Duro, 2005: 137-138).

Silencio y olvido se vinculan en el proceso (re)fundacional y adquieren particular importancia como ficción iniciática, cuyo valor ambiguo oscila entre el vacío - lo que no es - y el olvido - lo voluntariamente descartado y que, por lo mismo, tal vez sea más significante que lo efectivamente enunciado-.

Esta concepción de la realidad en términos de lengua, es decir, como materia narrada, encierra también una particular visión de la ficción y la realidad, ya que en la medida en que la realidad es básicamente discursiva, se desdibujan las fronteras entre estas dos categorías. La palabra se vuelve prácticamente un elemento ritual que permite la trasposición de un plano a otro, tal como se manifiesta cuando Elin intenta evadir(se) (d)el final trágico que le espera profiriendo las palabras «iNo es verdad! ¡No es verdad...!» (NEV: 1301), como si la palabra adquiriera un valor performativo capaz de transformar la realidad.

En efecto, en esta obra se ve claramente cómo la dicotomía realidad/ ficción es entendida en términos de verdad/mentira de modo tal que al desdibujarse las fronteras de las primeras dos también lo hacen las de las segundas, desbancando el mito de la verdad única y monolítica. Desde el punto de vista discursivo esta verdad inmutable encuentra su paralelo en la Historia, entendida como discurso oficial — bajo los paradigmas de cientificidad y objetividad en los que se ampara-. Al poner sobre la mesa el problema del estatuto lingüístico de la realidad, Nieva va mucho más allá del simple retruécano para proponer una verdadera filosofía del lenguaje como herramienta revolucionaria, en un sentido amplio, como apunta Aggor (2000: 435):

Language, in this context, becomes a key instrument of political power; in liberating it from regulation, the playwright counteracts language's quality as an agent of cultural domination. 
De este modo, la literatura de Nieva cuestiona los parámetros del discurso hegemónico, para proponer la posibilidad de la reescritura al discurso oficial (Imbert, 1990: 16-17), con un valor diametralmente opuesto a la reescritura propuesta desde el olvido tal como veíamos en $C D D$.

Esto supone la destrucción de la lógica binaria donde no hay más que una verdad a la que solo puede oponérsele la mentira. La lógica aquí propuesta admite los distintos niveles de verdad de manera reconciliable y, por lo mismo, las distintas capas que, aun negándose entre sí, conforman la historia como discurso.

Esta visión de la historia en capas se vincula también con la construcción de las obras como pequeños universos que pueden superponerse unos sobre otros para conformar un relato mayor del que no se dará cuenta, sino en la medida en que cada espectador pueda - o quiera - realizar esta síntesis en un nivel superior de lectura que englobe todas las demás. Esto se ve con claridad en la obras que conforman la trilogía representada en el festival de Avignon bajo el título Le retable des damnées ${ }^{11}$. El simple hecho de haberlas reunido para una representación colectiva da cuenta de una afinidad que solo puede explicarse en un metanivel superior; pequeñas piezas participando las unas en las otras de modo tal que todas pueden ser vistas como variantes (metáforas) de una historia mayor que podríamos denominar «macrohistoria».

Aun así, es importante entender que Nieva no propone una lógica discursiva directamente vinculada al contexto fascista, sino una perspectiva mucho más laxa de crítica a la construcción del sentido unívoco y, en lo que respecta a la palabra y al discurso, de la construcción de relatos totalizadores y totalitarios. Ni siquiera sería válido suponer una intención, en un sentido consciente, sino tan solo una semiótica del discurso que encuentra una explicación contextual más que pertinente, porque como bien señala Nieva (1980: 108): «[A]tentar contra una palabra es atentar contra un determinado sistema que se vale de ella».

\section{CONCLUSIÓN: EL COMPROMISO «OBLICUO»}

Uno de los fenómenos más interesantes de la literatura de este periodo es la tendencia — que creemos que puede ser considerada general- a una escritura en la que parecen ausentes referencias de tipo sociopolítico o co-

11 Estas tres obras también fueron publicadas conjuntamente en París por la editorial Actes SudPapiers en 1995 (Peña, 2001b: 481-482). 
nexiones directas más generales con la realidad de la época. Al contrario de lo que cabría esperar una vez superada la censura, momento en el que podría realizarse una fuerte crítica social y practicar una literatura de denuncia, ocurre un fenómeno curioso que es el de cierta tendencia a la evasión ${ }^{12}$.

Pero también se vuelve extraño considerar que en los procesos de transición y consolidación, en el que se forjan estos nuevos significados, no haya existido un interés en la literatura por ir ganando terreno - a nivel simbólico y a nivel real - a la censura que progresivamente se iba debilitando y cediendo espacios «nuevos». De ahí que llame la atención una producción que, aparentemente, no reclama la restitución de ciertos espacios que le son propios. No obstante, resulta patente que esta impresión está condicionada por modelos del arte que responden al paradigma del realismo literario.

El proceso de reapertura democrática implicó una revisión de los modelos semióticos de representación de los referentes culturales (desde la configuración simbólica de nación hasta la propia identidad del individuo). En este sentido, la transición es antes que nada una crisis simbólica. De ahí que la literatura como forma «institucionalizada» en la producción de sentidos sea un medio especialmente idóneo para canalizar esta experiencia de la crisis del sujeto.

Las últimas producciones de Francisco Nieva nos presentan un modelo sumamente alegórico en sus formas de vincularse a los procesos sociopolíticos. De hecho, sugiere claves fragmentadas de lectura, en las que las obras se constituyen como una gran alegoría del cambio y de la trasformación identitaria. Para ello se requiere un lector/espectador comprometido, en la medida en que la obra no orienta la lectura hacia este nivel. «Siempre es una falta la falta de memoria» dice Anaís en TQZ, y desata uno de los temas más polémicos de la crisis identitaria española.

Lo que sí se tratan son los grandes temas del proceso de regeneración identitaria: la nueva organización social, la exclusión de grupos minoritarios, el nuevo papel de la mujer en la sociedad, el valor simbólico del silencio y el problema de la memoria obstruida en la construcción identitaria. Pero nada es explícito. Situar al espectador en la zona oscura donde la contradicción queda suspendida sin resolución para obligarlo a completar el

12 Sobre el fenómeno de la «evasión» y la ausencia de compromiso en la literatura postfranquista véase Miralles (1980: 7-10). 
sentido desde una opción que supone siempre algún tipo de compromiso ético es, probablemente, uno de los vínculos más fuertes que la literatura nieviana realiza con la realidad de su tiempo.

\section{REFERENCIAS BIBLIOGRÁFICAS}

AGGOR, K. (1998). «Francisco Nieva's Nosferatu and the Theater of Cruelty». Revista Hispánica Moderna 52.2, 391-405.

- (2000). «Francisco Nieva, postmodern playwright». Hispanic Review $68.4,429-452$.

- (2009). Francisco Nieva y el teatro posmodernista. Madrid: Fundamentos.

BARRAJÓN, J. M. (1994). «Sobre la clasificación del teatro de Francisco Nieva». Ínsula 566, 5-6.

BUERO VALLEJO, A. (1960). «Obligada precisión acerca del 'imposibilismo'». Primer Acto 15, 1-6.

GARCÍA BARRIENTOS, J. L. (2001). Cómo se comenta una obra de teatro. Madrid: Síntesis.

GONZÁLEZ, A. (1980). «Introducción». En Maldita sean Coronada y sus hijas. Delirio del amor hostil. Madrid: Cátedra.

GONZÁLEZ DURO, E. (2005). La sombra del General. Qué queda del franquismo en España. Barcelona: Debate.

HUNTINGTON, S. P. (1994). La tercera ola: la democratización a finales del siglo XX. Barcelona: Paidós.

HUTCHEON, L. (1988). A Poetics of Postmodernism: History, Theory, Fiction. New York: Routledge.

IMBERT, G. (1990). Los discursos del cambio. Imágenes e imaginarios sociales en la España de la Transición (1976-1982). Madrid: Akal.

MIRALLES, A. (1980). «La peripecia del desencanto en el teatro español: la culpa es de todos y de ninguno». Estreno 4.2, 7-10.

NIEVA, F. (1976). «Pequeña teoría sobre un teatro histórico-didáctico». En Sombra y quimera de Larra, 5-28. Madrid: Fundamentos.

- (1980). «Breve poética teatral». En Maldita sean Coronada y sus hijas. Delirio del amor hostil, A. González (ed.), 93-117. Madrid: Cátedra. 
NIEVA, F. (1991). «Nota del autor». En Teatro completo, vol. 1, 173. Castilla-La Mancha: Servicio de Publicaciones de la Junta de Comunidades de Castilla-La Mancha.

- (2007). «Nieva ve a Penélope Cruz como protagonista de su revisada Catalina del demonio». El Mundo, 21/5, http://www.elmundo.es/elmundo/2007/03/21/cultura/1174496073.html.

PEÑA, J. F. (2001a). El teatro de Francisco Nieva. Vol. 1. Madrid: Universidad de Alcalá de Henares.

- (2001b). El teatro de Francisco Nieva. Vol. 2. Madrid: Universidad de Alcalá de Henares.

PÉREZ COTERILLO, M. (1975). «Introducción». Teatro furioso. Madrid: Akal/Ayuso.

PÉREZ-DÍAZ, V. (1993). La primacía de la sociedad civil: el proceso de formación de la España democrática. Madrid: Alianza.

- (1997). La esfera pública y la sociedad civil. Madrid: Taurus.

SASTRE, A. (1960a). «Teatro imposible y pacto social». Primer Acto 14, 1-2.

- (1960b). «A modo de respuesta». Primer Acto 16, 1-2.

VILLORIA MENDIETA, M. (1999). «El papel de la burocracia en la transición y consolidación de la democracia española: primera aproximación». Revista Española de Ciencia Política 1(1), 97-125.

YSÀS, P. (2004). Disidencia y subversión. La lucha del régimen franquista por su supervivencia, 1960-1975. Barcelona: Crítica.

WYNANTS, S. (2006). «Las reglas de juego de la democracia española». En La democracia española: realidades y desafíos. Análisis del sistema político español, M. Barreda y R. Borge (eds.), 67-227. Barcelona: UOC.

Recibido el 24 de junio de 2013.

Aceptado el 19 de septiembre de 2013. 
